\title{
Comparison of Pre-Attentive Auditory Discrimination at Gross and Fine Difference between Auditory Stimuli
}

\author{
Himanshu Kumar Sanju ${ }^{1}$ Prawin Kumar ${ }^{1}$ \\ ${ }^{1}$ Department of Audiology, All India Institute of Speech and Hearing, \\ Mysore, Karnataka, India \\ Address for correspondence Himanshu Kumar Sanju, MD, \\ Department of Audiology, AllSH, Mysore, Karnataka, India 570006 \\ (e-mail: himanshusanjuaiish@gmail.com).
}

Int Arch Otorhinolaryngol 2016;20:305-309.

\begin{abstract}
Introduction Mismatch Negativity is a negative component of the event-related potential (ERP) elicited by any discriminable changes in auditory stimulation.

Objective The present study aimed to assess pre-attentive auditory discrimination skill with fine and gross difference between auditory stimuli.

Method Seventeen normal hearing individual participated in the study. To assess preattentive auditory discrimination skill with fine difference between auditory stimuli, we recorded mismatch negativity (MMN) with pair of stimuli (pure tones), using / $1000 \mathrm{~Hz}$ / and $/ 1010 \mathrm{~Hz} /$ with $/ 1000 \mathrm{~Hz} /$ as frequent stimulus and $/ 1010 \mathrm{~Hz} /$ as infrequent stimulus. Similarly, we used $/ 1000 \mathrm{~Hz} /$ and $/ 1100 \mathrm{~Hz} /$ with $/ 1000 \mathrm{~Hz} /$ as frequent stimulus and $/ 1100 \mathrm{~Hz}$ as infrequent stimulus to assess pre-attentive auditory discrimination skill with gross difference between auditory stimuli. The study included 17 subjects with informed consent. We analyzed MMN for onset latency, offset latency, peak latency, peak amplitude, and area under the curve parameters.

Result Results revealed that MMN was present only in $64 \%$ of the individuals in both

Keywords

- auditory evoked potential

- event related potential

- attention conditions. Further Multivariate Analysis of Variance (MANOVA) showed no significant difference in all measures of MMN (onset latency, offset latency, peak latency, peak amplitude, and area under the curve) in both conditions.

Conclusion The present study showed similar pre-attentive skills for both conditions: fine $(1000 \mathrm{~Hz}$ and $1010 \mathrm{~Hz})$ and gross $(1000 \mathrm{~Hz}$ and $1100 \mathrm{~Hz})$ difference in auditory stimuli at a higher level (endogenous) of the auditory system.
\end{abstract}

\section{Introduction}

Electrophysiological measures are one of the objective modes of assessment to check the functioning of the auditory function. These measures complement the information provided by the behavioral measures, which are Differential Limen of Frequency (DLF) and Differential Limen of Intensity. An auditory evoked potential assessment with electrophysiological measures describes as series of electrical changes occurring in the peripheral and central nervous system,

received

July 4, 2015

accepted

September 14, 2015

published online

December 8, 2015 usually related to the sensory pathways. The auditory evoked potential is further classified as endogenous and exogenous. The exogenous potentials are primarily evoked by some external event-related to the dimension of the stimulus. Studies considered the possibility of studying auditory discrimination using event-related potentials. ${ }^{1,2}$ The endogenous potentials, similar to mismatch negativity (MMN), are responses resulting from internal events, such as cognition or perception. The mismatch negativity (MMN) is a component of event-related potential that has been extensively used to
Copyright $\odot 2016$ by Thieme Publicações License terms Ltda, Rio de Janeiro, Brazil
DOI http://dx.doi.org/

10.1055/s-0035-1570071. ISSN 1809-9777.

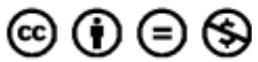


study the pre-attentive auditory discrimination skill and storage of regularities in stimulus features. ${ }^{3}$

Pre-attentive processing is the unconscious accumulation of information from the environment. All available information is pre-attentively processed. Then, our brain filters and processes the important information. We select information that has the highest salience (a stimulus that stands out the most) or relevance to what we are thinking about for a further and more complete analysis through conscious (attentive) processing. ${ }^{4,5}$ Our auditory system plays a very important role in collecting information for pre-attentive processing. When auditory stimuli or soundwaves strike the eardrum, they send a message to the brain via auditory nerves for preattentive processing. The skill to adequately filter information from pre-attentive auditory processing to attentive auditory processing is important for normal development. ${ }^{6}$ For acoustic pre-attentive auditory processing, the temporal cortex is the main site of activation; however, recent literature and research also showed the involvement of the frontal cortex. ${ }^{7,8}$ Literature also reports that detection of slight variation in complex musical pattern activates the right ventromedial prefrontal cortex. $^{7}$

Mismatch Negativity (MMN) was first described by Naatanen et al. in $1978 .{ }^{9}$ Our brain is able to perceive even a minute change in the acoustic environment, which can be in terms of intensity (loudness), phase, frequency (pitch), and location of auditory stimuli. MMN has been gaining importance as a measure to assess discrimination (i.e., minute change) in the acoustic environment. Naatanen and Escera defined MMN as "an electric brain response, a negative component of the event-related potential (ERP), elicited by any discriminable change (deviant) in some repetitive aspect of auditory stimulation (standard), usually peaking at around $100-200 \mathrm{~ms}$ from onset." ${ }^{10}$ Mismatch Negativity is a negative component of event-related potential (ERP) elicited by any discriminable changes in auditory stimulation (Naatanen \& Alho, 1997) ${ }^{11}$ MMN can be evoked even in the absence of attention and is easy to administer. ${ }^{12}$ It can occur when the difference between the standard and deviant stimuli is as small as $8 \mathrm{~Hz}$, or even when stimulus differences are near psychophysical threshold. ${ }^{13}$ Sams et al. also showed that MMN were present when deviant stimuli were barely discriminable from the standard stimuli, even though the differences were not perceptible. ${ }^{14} \mathrm{MMN}$ reflects the central code of stimulus change; its amplitude and latency relate to the degree to which deviant stimuli differ from standard stimuli, not to absolute levels of deviant/standard stimuli. Thus, MMN is considered an objective neurophysiological test of auditory discrimination. Moreover, it appears that MMN reflects neuronal representation of the discrimination of numerous auditory stimulus attributes. If MMN reflects the ability to discriminate between acoustic stimuli, it is of clinical importance as well, since speech perception inherently depends on neuronal responses to changes in stimulus. ${ }^{15}$ Based on the literature, we observed that there is a dearth of literature that compares pre-attentive auditory discrimination skill with fine and gross difference of acoustic stimuli. Therefore, the present study aims to fill this gap.

\section{Method}

\section{Participants}

Seventeen normal hearing individuals from a private science college participated in the study with informed consent. Their age range was $18-25$ years (mean age 21.5 years).

\section{Participant Selection Criteria}

All the participants had normal hearing thresholds as defined by pure tone thresholds of $<15 \mathrm{dBHL}$ at $250 \mathrm{~Hz}$ to $8000 \mathrm{~Hz}$. Further, they had normal middle ear function as revealed by the middle ear analyzer. Participants presenting any other otological, neuromuscular, or neurological problem were excluded from the study.

\section{Testing Environment}

We performed electrophysiological tests in a sound treated room where the noise level was as per the guidelines in ANSI S3.1 (1999). The testing rooms were well lit and air-conditioned for the comfort of the examiner, as well as the participant.

\section{Instrumentation}

Calibrated double-channel clinical audiometer Orbiter 922 (MADSEN-GN Otometrics, Denmark) was used for pure tone audiometry. Calibrated GSI Tympstar immittance meter (Grason-Stadler, U.S.A) was used for tympanometry and reflexometry. We used the Intelligent Hearing System (Miami, USA) with smart EP to record Mismatch Negativity.

\section{Procedure}

We obtained pure tone thresholds using a modified version of the Hughson and Westlake procedure across octave frequencies from 250 to $8000 \mathrm{~Hz}$ for air conduction and 500, 1000, 2000, and $4000 \mathrm{~Hz}$ for bone conduction. The middle ear analyzer (GSI-Tympstar) was used to carry out the tympanometry using a probe tone frequency of $226 \mathrm{~Hz}$ and to obtain ipsilateral and contralateral acoustic reflex thresholds at 500, 1000,2000 , and $4000 \mathrm{~Hz}$. To assess pre-attentive auditory discrimination skill with fine difference between auditory stimuli, we recorded Mismatch negativity (MMN) with pairs of stimuli (pure tones). We used $/ 1000 \mathrm{~Hz} /$ and $/ 1010 \mathrm{~Hz} /$ with $/ 1000 \mathrm{~Hz} /$ as frequent stimulus and $/ 1010 \mathrm{~Hz} /$ as infrequent stimulus. Similarly, /1000 Hz/ and $/ 1100 \mathrm{~Hz} /$ with $/ 1000 \mathrm{~Hz} /$ as frequent stimulus and $/ 1100 \mathrm{~Hz} /$ as the infrequent stimulus, were used to assess pre-attentive auditory discrimination skill with gross difference between auditory stimuli. The total duration of the stimuli was 200 milliseconds with 30 milliseconds of rise-fall and a plateau of 140 milliseconds. We emitted the stimuli aided by the Aux Viewer program. We converted the wave file to a stimulus file for AEPs using the Stimconv software (Intelligent Hearing System, Miami, U.S.A.). We then recorded MMN in a vertical montage with ' $F z$ ' as the positive electrodes referenced to the nape of the neck. We placed the ground electrode on the lower forehead. A second channel recorded eye blink response. The sweeps with large eye blink artifacts were eliminated from the averaging. Stimuli were presented in the oddball 
paradigm with the probability of standard and deviant stimulus of $80 \%$ and $20 \%$ at $70 \mathrm{dBnHL}$, respectively. We presented stimuli in the rarefaction polarity with a repetition rate of $1.1 /$ second. The responses were averaged for 150 sweeps (150 infrequent stimuli + the corresponding number of frequent stimuli) from -50 to 500 milliseconds (with reference to stimulus onset). The band pass filter was set to the frequency range of 0.1 to $30 \mathrm{~Hz}$, while amplified up to 50,000 times. We presented stimuli binaurally. Participants sat comfortably to avoid muscular artifacts and watched a silent movie to promote passive listening. The subjects were instructed not to pay attention to the auditory stimuli. We cleaned and placed disc electrodes on the skin surface of the target electrode sites. The absolute impedance was less than $5 \mathrm{k} \Omega$ and inter-electrode impedance was less than $2 \mathrm{k} \Omega$ while recording MMN. Aside from recording MMN in the conventional paradigm for each stimulus pair, we also recorded LLRs (Long Latency Responses) for the infrequent stimulus for 150 presentations, keeping the same recording parameter used for MMN.

\section{Response Analysis}

We obtained pure tone thresholds using a modified version of the Hughson and Westlake procedure across octave frequencies from 250 to $8000 \mathrm{~Hz}$ for air conduction and 500, 1000, 2000, and $4000 \mathrm{~Hz}$ for bone conduction. The middle ear analyzer (GSITympstar) was used to carry out the tympanometry using a probe tone frequency of $226 \mathrm{~Hz}$ and to obtain ipsilateral and contralateral acoustic reflex thresholds at 500,1000, 2000, and $4000 \mathrm{~Hz}$. To assess pre-attentive auditory discrimination skill with fine difference between auditory stimuli, we recorded Mismatch negativity (MMN) with pairs of stimuli (pure tones). We used $/ 1000 \mathrm{~Hz} /$ and $/ 1010 \mathrm{~Hz} /$ with $/ 1000 \mathrm{~Hz} /$ as frequent stimulus and $/ 1010 \mathrm{~Hz} /$ as infrequent stimulus. Similarly, $/ 1000 \mathrm{~Hz}$ / and $/ 1100 \mathrm{~Hz} /$ with $/ 1000 \mathrm{~Hz}$ / as frequent stimulus and $/ 1100 \mathrm{~Hz} /$ as the infrequent stimulus, were used to assess pre-attentive auditory discrimination skill with gross difference between auditory stimuli. The total duration of the stimuli was 200 milliseconds with 30 milliseconds of rise-fall and a plateau of 140 milliseconds. We emitted the stimuli aided by the Aux Viewer program. We converted the wave file to a stimulus file for AEPs using the Stimconv software (Intelligent Hearing System, Miami, U.S.A.). We then recorded MMN in a vertical montage with 'Fz' as the positive electrodes referenced to the nape of the neck. We placed the ground electrode on the lower forehead. A second channel recorded eye blink response. The sweeps with large eye blink artifacts were eliminated from the averaging. Stimuli were presented in the oddball paradigm with the probability of standard and deviant stimulus of $80 \%$ and $20 \%$ at $70 \mathrm{dBnHL}$, respectively. We presented stimuli in the rarefaction polarity with a repetition rate of $1.1 /$ second. The responses were averaged for 150 sweeps (150 infrequent stimuli + the corresponding number of frequent stimuli) from -50 to 500 milliseconds (with reference to stimulus onset). The band pass filter was set to the frequency range of 0.1 to $30 \mathrm{~Hz}$, while amplified up to 50,000 times. We presented stimuli binaurally. Participants sat comfortably to avoid muscular artifacts and watched a silent movie to promote passive listening. The subjects were instructed not to pay attention to the auditory stimuli. We cleaned and placed disc electrodes on the skin surface of the target electrode sites. The absolute impedance was less than $5 \mathrm{k} \Omega$ and inter-electrode impedance was less than $2 \mathrm{k} \Omega$ while recording MMN. Aside from recording MMN in the conventional paradigm for each stimulus pair, we also recorded LLRs (Long Latency Responses) for the infrequent stimulus for 150 presentations, keeping the same recording parameter used for MMN.

\section{Waveform Analysis}

For the identification of the MMN true response through visual detection, MMN should be the first negative trough in the latency range of the $\mathrm{N}_{1}-\mathrm{P}_{2}$ or $\mathrm{P}_{2}-\mathrm{N}_{2}$ complex of LLR of amplitude greater than $-0.3 \mu \mathrm{V}$ and the positive peak should follow the negative peak. If the extra negativity occurred in the $P_{1}$ area, it was ignored.

To analyze the data collected from musicians and nonmusicians, we extracted the following response measures from the MMN for each participant:

i. Onset Latency: It is the time, in milliseconds, when negativity started in the subtracted waveform.

ii. Offset Latency: It is the time, in milliseconds, when the negativity reached the baseline activity in the subtracted waveform.

iii. Peak Latency: It is the time, in milliseconds, at which negativity reached its peak in the subtracted waveform.

\section{Statistical Analysis}

We used descriptive statistics to find out mean and standard deviation (SD) for all the parameters of MMN (onset latency, offset latency, peak latency, peak amplitude, and area under the curve). We conducted a Multivariate Analysis of Variance (MANOVA) to compare each measure (onset latency, offset latency, peak latency, peak amplitude, and area under the curve) under two conditions: gross difference in auditory stimuli and fine difference in auditory stimuli.

\section{Result}

To analyze the data collected, we conducted a descriptive statistics and a Multivariate Analysis of Variance (MANOVA). Out of 17 individuals, MMN was present in only 11 of them (64\%). Descriptive statistics showed similar outcomes for both the condition shown in - Table 1 for onset, offset, and peak latency and that shown in - Table $\mathbf{2}$ for peak amplitude and area under the curve. We extracted the different measures of MMN, such as onset latency, offset latency, peak latency, peak amplitude, and area under curve from the MMN waveform through visual inspection for all 11 participants. We applied MANOVA to compare between two conditions (gross and fine) for each measure of MMN. Sample waveforms of mismatch negativity is shown in - Fig. $\mathbf{1}$.

\section{Onset Latency, Offset Latency, and Peak Latency}

MANOVA showed no significant difference between both conditions, fine $(/ 1000 \mathrm{~Hz} /$ and $/ 1010 \mathrm{~Hz} /)$ and gross $(/ 1000$ $\mathrm{Hz} /$ and $/ 1100 \mathrm{~Hz} /)$ in terms of onset latency $[\mathrm{F}(1,20)=0.77$; 
Table 1 Mean and standard deviation (SD) of onset latency, offset latency, and peak latency for the fine and gross conditions

\begin{tabular}{|l|l|l|l|l|l|l|}
\hline \multirow{2}{*}{ Parameters } & \multicolumn{2}{|l|}{ Onset Latency (ms) } & \multicolumn{2}{l|}{ Offset Latency (ms) } & \multicolumn{2}{l|}{ Peak Latency (ms) } \\
\cline { 2 - 7 } & Mean & SD & Mean & SD & Mean & SD \\
\hline Fine Difference & 161.54 & 28.02 & 287.72 & 23.86 & 213.27 & 24.36 \\
\hline Gross Difference & 173.36 & 34.65 & 261.54 & 37.63 & 216.45 & 37.16 \\
\hline
\end{tabular}

Table 2 Mean and standard deviation (SD) of peak amplitude and area under curve for the fine and gross conditions

\begin{tabular}{|l|l|l|l|l|}
\hline Parameters & Peak Amplitude $(\mu \mathrm{v})$ & \multicolumn{2}{l|}{ Area under Curve $(\mu \mathrm{V} \mu \mathrm{sec})$} \\
\hline Groups & Mean & SD & Mean & SD \\
\hline Fine Difference & 2.63 & 1.08 & 176.17 & 55.14 \\
\hline Gross Difference & 2.93 & 0.84 & 131.58 & 45.77 \\
\hline
\end{tabular}

$\left.\mathrm{p}>0.05 ; \eta^{2}=0.03\right]$, offset latency $[\mathrm{F}(1,20)=3.79 ; \mathrm{p}>0.05$ $\left.\eta^{2}=0.16\right]$, and peak latency $\left[\mathrm{F}(1,20)=0.05 ; \mathrm{p}>0.05 ; \eta^{2}=0.00\right]$.

\section{Peak Amplitude and Area under Curve}

MANOVA showed no significant difference between both conditions, fine $(/ 1000 \mathrm{~Hz} /$ and $/ 1010 \mathrm{~Hz} /)$ and gross $(/ 1000$ $\mathrm{Hz} /$ and $/ 1100 \mathrm{~Hz} /$ ) difference in terms of peak amplitude $\left[\mathrm{F}(1,20)=0.52 ; \mathrm{p}>0.05 ; \eta^{2}=0.02\right]$ and area under curve $\left[\mathrm{F}(1,20)=4.25 ; \mathrm{p}>0.05 ; \eta^{2}=0.17\right]$.

\section{Discussion}

The present study showed no significant difference in measures of mismatch negativity in both the fine condition (/1000 $\mathrm{Hz} /$ and $/ 1010 \mathrm{~Hz} /)$ as well as gross condition $(/ 1000 \mathrm{~Hz} /$ and $/ 1100 \mathrm{~Hz} /$ ), which showed similar auditory discrimination skill at pre-attentive level (discrimination skill without giving attention). Lang, Nyrke, Aaltonen, Raimo, and Naatanen recorded MMN to a frequency deviance and correlated it to a behavioral discrimination task. ${ }^{16}$ They divided their participants into three groups (good, poor, and intermediate) based on their performance in the behavioral discrimination task. They found that MMN was present for a smaller frequency deviance (fine) in the good performers group. On the other hand, in the group of poor performers, MMN was elicited with a greater frequency deviance (gross). ${ }^{16}$ Sams et al also reported that MMN were present when the deviant stimuli were just discriminable from the standard stimuli, even though the differences were not perceptible. ${ }^{14}$ According to Kraus et al., MMN can occur even when the difference between the standard and deviant stimuli is as small as $8 \mathrm{~Hz}$ or even when stimulus differences are near psychophysical threshold. ${ }^{13}$ The present study is in contrast with the study done by Picton et al, which stated that MMN reflect the central code of stimulus change (i.e., its amplitude increases and latency decreases with increase in the difference

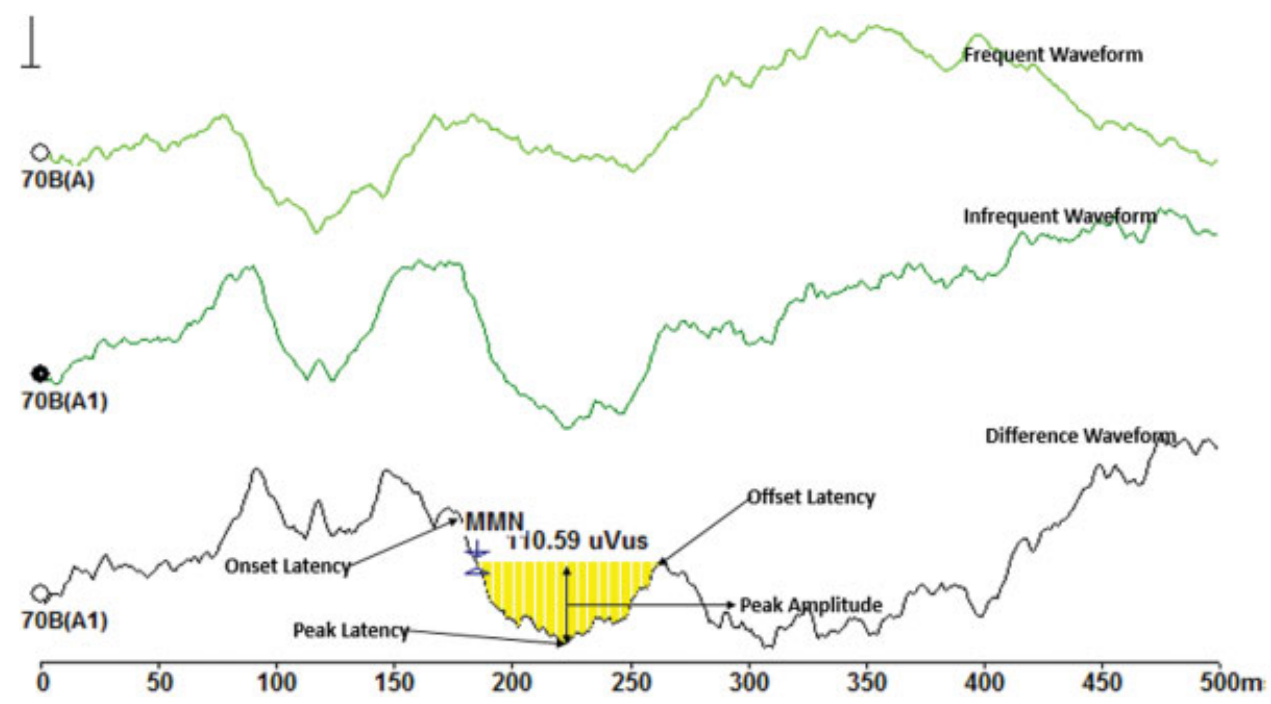

Fig. 1 Sample waveform of mismatch negativity. 
between standard and deviant stimuli). Generally, the larger the difference between standard and deviant stimuli, the earlier the latency and larger the amplitude of MMN, although there may be a ceiling effect in amplitudes with a larger difference. ${ }^{17}$ This studýs outcomes showed that, at the pre-attentive level of auditory processing, the processing of auditory stimuli (infrequent), which differs slightly from the other (frequent), is almost similar when compared with the processing of auditory stimuli (infrequent), which differs grossly from other (frequent). Thus, we may conclude there is no difference in pre-attentive auditory discrimination when the infrequent differs slightly from frequent or when it differs grossly from frequent. The current study highlights that MMN was present in only $64 \%$ of the normal population. Therefore, the finding of MMN in clinical population should be interpreted cautiously, as there is a chance of absence of MMN even in normal hearing individuals.

\section{Conclusion}

The present study showed that our auditory system has similar auditory discrimination skill with gross as well as fine difference in auditory stimuli at pre-attentive level (discrimination without attention).

\section{References}

1 Ceponiene R, Kushnerenko E, Fellman V, Renlund M, Suominen K, Näätänen R. Event-related potential features indexing central auditory discrimination by newborns. Brain Res Cogn Brain Res 2002;13(1):101-113

2 Chang M, Iizuka H, Naruse Y, Ando H, Maeda T. Unconscious learning of auditory discrimination using mismatch negativity (MMN) neurofeedback. Sci Rep 2014;4:6729

3 Paavilainen P. The mismatch-negativity (MMN) component of the auditory event-related potential to violations of abstract regularities: a review. Int J Psychophysiol 2013;88(2):109-123
4 Atienza M, Cantero JL, Escera C. Auditory information processing during human sleep as revealed by event-related brain potentials. Clin Neurophysiol 2001;112(11):2031-2045

5 Van AH. Two stages in visual information processing and visual perception? Vis Cogn 1996;3(4):325-362

6 Seri S, Pisani F, Thai JN, Cerquiglini A. Pre-attentive auditory sensory processing in autistic spectrum disorder. Are electromagnetic measurements telling us a coherent story? Int J Psychophysiol 2007;63(2):159-163

7 Habermeyer B, Herdener M, Esposito F, et al. Neural correlates of pre-attentive processing of pattern deviance in professional musicians. Hum Brain Mapp 2009;30(11):3736-3747

8 Klamer D, Svensson L, Fejgin K, Pålsson E. Prefrontal NMDA receptor antagonism reduces impairments in pre-attentive information processing. Eur Neuropsychopharmacol 2011;21(3):248-253

9 Näätänen R, Gaillard AW, Mäntysalo S. Early selective-attention effect on evoked potential reinterpreted. Acta Psychol (Amst) 1978;42(4):313-329

10 Näätänen R, Escera C. Mismatch negativity: clinical and other applications. Audiol Neurootol 2000;5(3-4):105-110

11 Näätänen R, Alho K. Mismatch negativity-the measure for central sound representation accuracy. Audiol Neurootol 1997;2(5): 341-353

12 Nätänen R, Paavilainen P, Tiitinen H, Jiang D, Alho K. Attention and mismatch negativity. Psychophysiology 1993;30(5):436-450

13 Kraus N, McGee T, Micco A, Sharma A, Carrell T, Nicol T. Mismatch negativity in school-age children to speech stimuli that are just perceptibly different. Electroencephalogr Clin Neurophysiol 1993; 88(2):123-130

14 Sams M, Paavilainen P, Alho K, Näätänen R. Auditory frequency discrimination and event-related potentials. Electroencephalogr Clin Neurophysiol 1985;62(6):437-448

15 Kraus N, McGee T, Carrell T, King C, Littman T, Nicol T. Discrimination of speech-like contrasts in the auditory thalamus and cortex. J Acoust Soc Am 1994;96(5 Pt 1):2758-2768

16 Lang H, Nyrke T, Aaltonen O, Raimo I, Näätänen R. Pitch discrimination performance and auditory event-related potentials. In C. $H$. M. Brunia, A. W. K. Gaillard, A. Kok, G. Mulder, \& M. N. Verbaten (Eds.), Psychophysiological brain research (Vol. 1). Tilburg: Tilburg University Press; 1990:294-298

17 Picton TW, Alain C, Otten L, Ritter W, Achim A. Mismatch negativity: different water in the same river. Audiol Neurootol 2000;5; (3-4):111-139 